\title{
Ankylosing Spondylitis Associated with Pulmonary Arterial Hypertension
}

\author{
Yao-Min Hung ${ }^{1,2}$, Chin-Chang Cheng ${ }^{2,3}$, Shue-Ren Wann ${ }^{1,2}$ and Shoa-Lin Lin ${ }^{4}$
}

\begin{abstract}
Pulmonary arterial hypertension (PAH) is a frequent complication of connective tissue diseases, such as systemic sclerosis, systemic lupus erythematosus and rheumatoid arthritis. However, the occurrence of PAH in a patient with ankylosing spondylitis (AS) has not been previously reported with a detailed clinical description in the English literature. We herein report the first case of AS associated PAH in a 27-year-old Taiwanese man with a chief complaint of intermittent palpitations lasting for two years. To the best of our knowledge, this is the first reported case of AS associated with PAH with a detailed clinical description and findings of right heart catheterization published in the English literature.
\end{abstract}

Key words: ankylosing spondylitis, pulmonary arterial hypertension, right-heart catheterization

(Intern Med 54: 431-434, 2015)

(DOI: 10.2169/internalmedicine.54.3160)

\section{Introduction}

According to the most updated definition and classification issued by the World Health Organization (WHO), the diagnosis of pulmonary hypertension $(\mathrm{PH})$ requires right heart catheterization. $\mathrm{PH}$ is confirmed when the mean pulmonary artery pressure is $\geq 25 \mathrm{mmHg}$ at rest. The $\mathrm{WHO}$ has classified $\mathrm{PH}$ into five groups based on the underlying mechanism $(1,2)$. Pulmonary arterial hypertension (PAH) includes a group of diseases that affect the small pulmonary arteries and is diagnosed based on a pulmonary capillary wedge pressure (PCWP) or left atrial pressure of $<15 \mathrm{~mm}$ $\mathrm{Hg}$ in the presence of $\mathrm{PH}$. Of all etiologies, connective tissue disease (CTD) is the most common condition associated with PAH. The onset of PAH in patients with CTD is common, with an incidence ranging from 0.5 to $15 \%$ according to the findings of right heart catheterization $(3,4)$. In cohort studies conducted in the USA and Europe, the majority of patients with PAH associated with CTD (CTD-PAH) have been found to have systemic sclerosis (5-7), whereas systemic lupus erythematosus (SLE) and mixed connectivetissue disease (MCTD) are more common in Japanese pa- tients with PAH (8).

Ankylosing spondylitis (AS) is a chronic, progressive, connective tissue disorder characterized by inflammation of the joints of the spine and sacroiliac region.

However, the concurrence of PAH in a patient with AS has rarely been described in the English literature $(9,10)$. According to a website search conducted on March 9, 2014 (11), 18,738 individuals with ankylosing spondylitis have been studied. Among them, $11(0.06 \%)$ were found to have pulmonary hypertension. However, none of the patients received right heart catheterization, and $\mathrm{PH}$ is not equal to PAH. We herein report a case of early-stage PAH detected via right heart catheterization following the exclusion of other known causes in a 27-year-old Taiwanese man with a 12-year history of AS.

\section{Case Report}

A 27-year-old man who had experienced intermittent palpitations for a decade was admitted to our hospital in August 2012. He complained of sporadic palpitations and had visited several hospitals within the past two years, being diagnosed with paroxysmal supraventricular tachycardia

\footnotetext{
${ }^{1}$ Department of Emergency Medicine, Kaohsiung Veterans General Hospital, Taiwan, ${ }^{2}$ School of Medicine, National Yang-Ming University, Taiwan, ${ }^{3}$ Cardiovascular Center, Kaohsiung Veterans General Hospital, Taiwan and ${ }^{4}$ Division of Cardiology, Kaohsiung Yuan's General Hospital, Taiwan

Received for publication April 28, 2014; Accepted for publication July 7, 2014

Correspondence to Dr. Shoa-Lin Lin, sllin@yuanhosp.com.tw
} 
(PSVT) attacks two to three times a year. According to his history, he began to visit our outpatient department at 15 years of age due to low back pain with limited motion of the lumbar spine and severe tenderness in the right buttock. He reported that the pain improved with exercise, with no improvements at rest. His family history revealed that his father had had AS for many years. The patient subsequently underwent a series of examinations, which showed a normal serum immunoglobulin level, negative antinuclear antibody (ANA) and rheumatoid factor (RF) titers and positive human leukocyte antigen (HLA)-B27 antigens. On a radiographic examination of the pelvis (Fig. 1), grade 2 bilateral sacroiliitis was diagnosed according to the modified New York classification (12). However, typical cervical and lumbar spine syndesmophytes were not identified. His visual acuity was normal, and no inflammation was noted in the anterior chamber of the eye fundus. He therefore received sul-

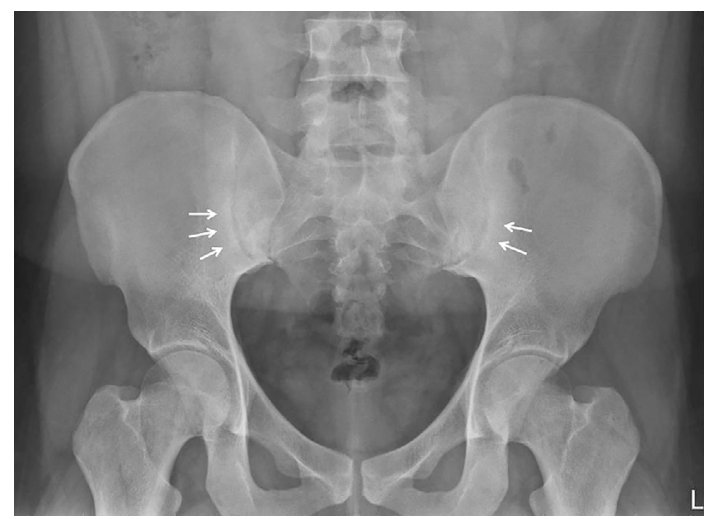

Figure 1. X-ray of the pelvis, depicting small areas of bilateral increased density and/or sclerosis (arrows) over the sacroiliac joints, indicating grade 2 bilateral sacroiliitis. fasalazine and nonsteroidal anti-inflammatory drugs (NSAIDs), with a fair response.

The patient denied any history of exposure to toxic chemicals or appetite suppressants during the course of therapy. There was no angina or exertional dyspnea. However, intermittent palpitations had occurred since 18 years of age when he drank coffee or experienced stress. The patient had been told that he had episodes of PSVT at several other hospitals. However, he visited our cardiovascular center in October 2011 and was followed up regularly. Since that time, he had experienced no more PSVT attacks. Nevertheless, he reported occasional lower back pain, although the episodes gradually decreased following analgesic treatment (sulfasalazine and meloxicam). Serial follow-up of inflammatory markers revealed a normal erythrocyte sedimentation rate (ESR) (11, 13 and $7 \mathrm{~mm} / \mathrm{hr}$ ) (normal range for men: 1-13 $\mathrm{mm} / \mathrm{hr}$ ) and C-reactive protein (CRP) level (0.44, 0.74 and $0.78 \mathrm{mg} / \mathrm{dL}$ ) (normal range: $<1.0 \mathrm{mg} / \mathrm{dL}$ ). Therefore, there was no evidence of high disease activity. On-site 12-lead electrocardiography (Fig. 2) showed right axis deviation, R/s $>1$ at V1 and inverted $\mathrm{T}$ waves over V1 to V4, which suggested right ventricular hypertrophy and strain. A chest film demonstrated no abnormal findings; however, transthoracic echocardiography (Fig. 3) revealed mild right ventricle (RV) hypertrophy, a dilated right atrium (RA), RV systolic dysfunction and dilated main pulmonary arteries. Furthermore, Doppler echocardiography disclosed mild pulmonary and tricuspid regurgitation, with an ejection fraction in the left ventricle of $64 \%$ and an estimated systolic pulmonary artery pressure (PAP) of approximately $43 \mathrm{mmHg}$ (determined via tricuspid regurgitation jet measurements). Admission for a further evaluation was suggested; however, the patient refused due to a planned trip to a foreign country. Therefore, oral propranolol was prescribed prn for palpitations and

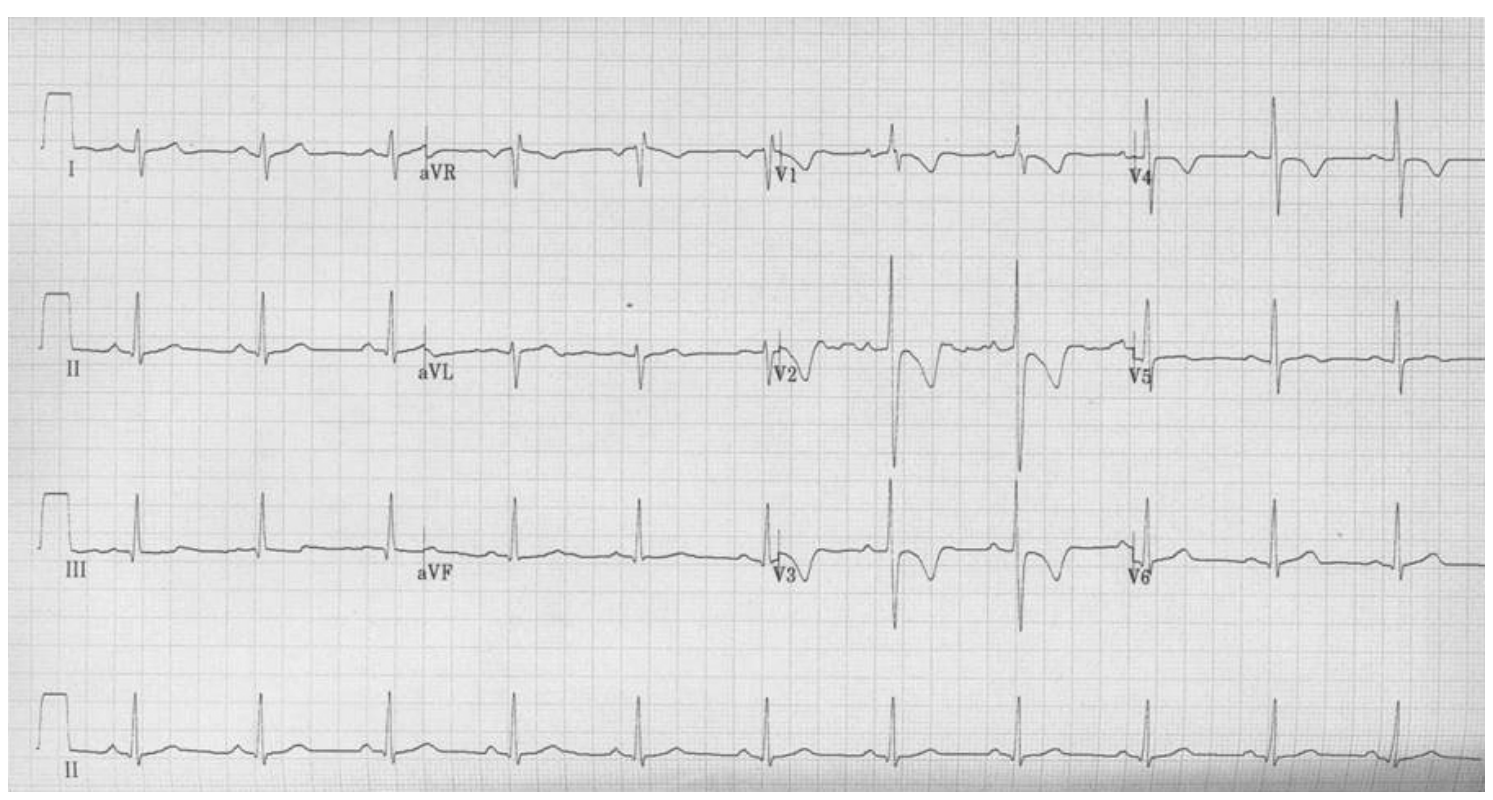

Figure 2. 12-lead electrocardiography showed inverted $T$ waves over the precordial leads, with right axis deviation and $\mathrm{R} / \mathrm{s}>1$ in $\mathrm{V1}$, suggesting right ventricular hypertrophy. 

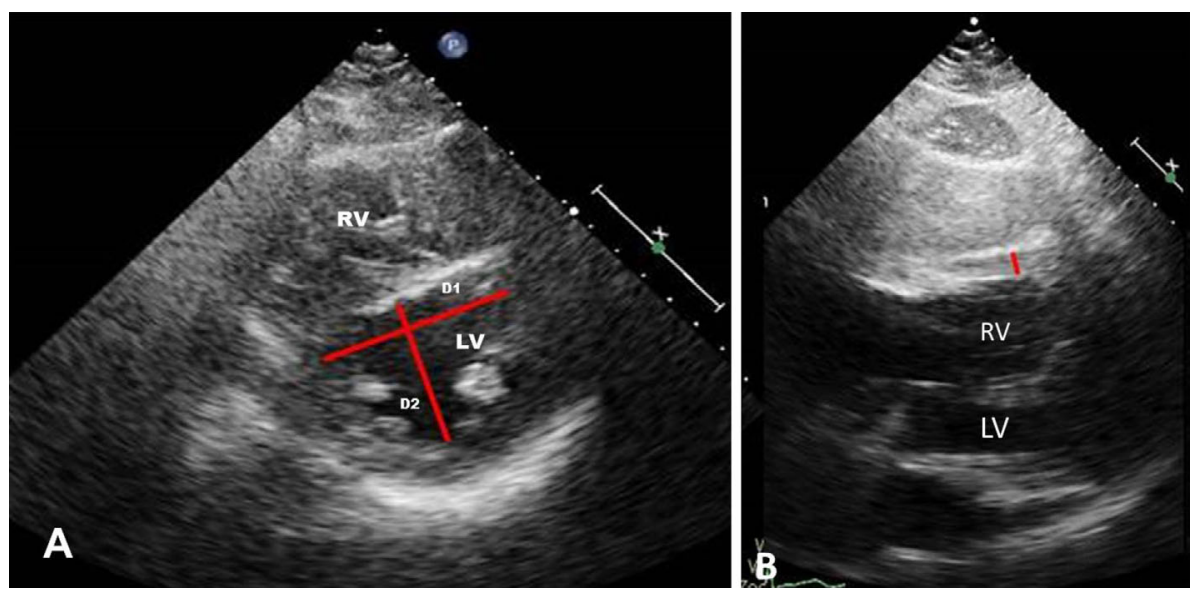

Figure 3. Two-dimensional echocardiography on the parasternal short-axis view disclosed flattening of the interventricular septum during the diastolic phase, with an eccentric index of $>1$ (D1=5.1 $\mathrm{mm} / \mathrm{D} 2=4.4 \mathrm{~mm}$ ), where D1 and D2 indicate the horizontal and longitudinal diameter (red line) of the left ventricle, respectively $(A)$. On the subcostal view, right ventricular hypertrophy $(\mathrm{RV}$ thickness $=6$ $\mathrm{mm}$, red line) is shown $(\mathrm{B})$.

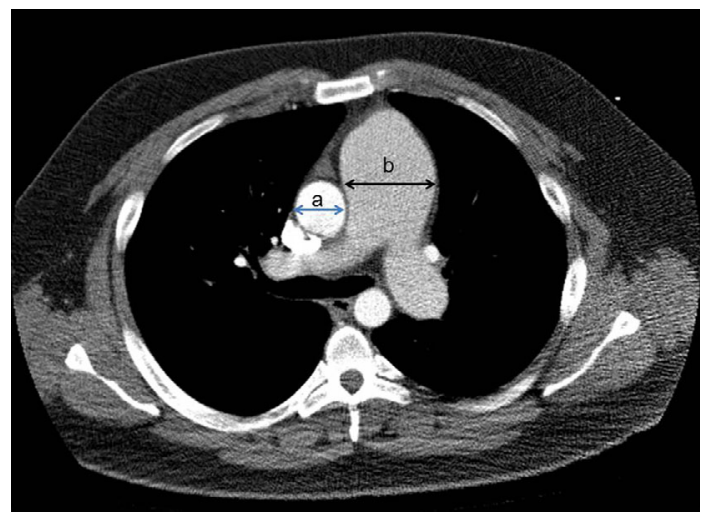

Figure 4. Chest computed tomography demonstrated no evidence of remarkable filling defects in major pulmonary arteries; however, the main pulmonary arteries were enlarged (4.9 cm) beyond the ascending aorta $(3.0 \mathrm{~cm})$. " $a$ " and " $b$ " indicate the diameter of the ascending aorta and main pulmonary artery, respectively.

\section{tachycardia.}

The patient returned to our department in June 2012 following several episodes of respiratory tract infection with prolonged coughing within the past six months. Pulmonary function tests showed a normal ventilatory function and negative response to methacholine. The oxygen saturation was $96 \%$ on room air according to pulse oximetry. An eosinophilic cationic protein (ECP) test showed a value of $5.81 \mathrm{ug} / \mathrm{L}$ (reference range: 2.3-15.9). Immunological analyses revealed high levels of Ig E in the blood (total Ig E: 150 $\mathrm{IU} / \mathrm{mL}$ (reference range: adult <87) with normal Ig G, Ig A and $\mathrm{Ig} \mathrm{M}$ values. A plasma D-Dimer assay showed a level of $383 \mathrm{ng} / \mathrm{mL}$ (reference range: $<500$ ), while the ESR was $21 \mathrm{~mm} / \mathrm{h}$ (reference range: M: 2-6, F: 2-12) and the highsensitivity CRP level was $0.782 \mathrm{mg} / \mathrm{dL}$ (reference range: < 0.8).

In August 2012, the patient was admitted for a further as- sessment. His body height and weight were $169 \mathrm{~cm}$ and 98 $\mathrm{kg}$, respectively The findings of physical examinations were unremarkable, except for a split second pulmonary heart sound. Routine biochemical parameters were within the normal limits, and there was no evidence of hepatic dysfunction or HIV infection. The plasma D-Dimer level was $631 \mathrm{ng} /$ $\mathrm{mL}$, and the B-type natriuretic peptide (BNP) level was 32 $\mathrm{pg} / \mathrm{mL}$ (reference range: $<100$ ). Chest computed tomography (Fig. 4) and lung perfusion scintigraphy showed no signs of pulmonary embolism or obstructive or restrictive pulmonary disease, and polysomnography showed no evidence of sleep apnea. Transesophageal echocardiography (TEE) revealed an intact interatrial septum without shunting in addition to a mildly dilated main pulmonary artery with mild RV hypertrophy, global RV hypokinesia and systolic dysfunction. Based on the above findings, a diagnosis of pulmonary arterial hypertension was suspected. A right cardiac catheterization study demonstrated the following findings: pulmonary capillary wedge pressure $=12 \mathrm{~mm} \mathrm{Hg}$, main pulmonary artery pressure $=78 / 44 \mathrm{mmHg}$ (mean: $55 \mathrm{mmHg}$ ), RV pressure $=70 / 10 \mathrm{mmHg}$, mean right atrial pressure $=13 \mathrm{mmHg}$, cardiac output $=7.14 \mathrm{~L} / \mathrm{min}$, cardiac index $=3.43 \mathrm{~L} / \mathrm{min}$ and main pulmonary artery oxygen saturation $=75 \%$. A consultation with a rheumatologist and serial tests showed negative findings for thyroid disorders and connective tissue diseases [ANA, ENA screening, anti-double-stranded DNA, anti-ribonucleoprotein (RNP) antibody Sm, SS-A/Ro, SS-B/ La, centromere, Scl-70, Venereal Disease Research Laboratory (VDRL) and rheumatoid factor were all negative]. Furthermore, abdominal sonography was negative, with no evidence of portal hypertension. The patient was therefore diagnosed with pulmonary arterial hypertension associated with AS. Exercise testing with the six-minute walk test (6MWT) was performed to evaluate his baseline functional status. Consequently, the initial 6MWT was completed within 385 meters, with a Borg dyspnea scale of 1 and 
WHO functional class of II. The maximum aerobic ability was approximately $43.74 \%$ of the predicted exercise limitation. The sulfasalazine treatment was continued and diltiazem therapy was initiated, and the patient was discharged with symptomatic improvement. In addition to diltiazem (30 $\mathrm{mg}$ tid), we requested coverage for bosentan from the National Health Insurance Bureau (NHIB), as a diagnosis of PAH was confirmed. Thereafter, treatment with bosentan at a dose of $62.5 \mathrm{mg}$ bid was initiated following approval from the NHIB, then subsequently titrated to $125 \mathrm{mg}$ bid. The patient has remained stable for eight months of follow-up, with a normal hemoglobin level and liver function parameters.

\section{Discussion}

Aortitis and periaortitis are important cardiovascular manifestations of AS. Inflammatory lesions progressing to fibrosis are primarily localized in the aortic root, which may cause aortic regurgitation in patients with AS (12). Doppler/ echocardiography can be used to easily detect the presence and severity of aortic insufficiency. The echocardiography findings (both transthoracic and transesophageal echocardiography) in the present case revealed an intact aortic valve without aortic insufficiency, and the patient presented with PSVT as the first symptom. Although he received a diagnosis of PSVT at other hospitals based on previous events, no such diagnosis was made at our hospital. Therefore, we considered the PSVT to be an independent condition unrelated to AS or PAH.

The occurrence of PAH is associated with rheumatoid arthritis, systemic lupus erythematosus, mixed connective tissue disease and even Sjögren's syndrome (13); however, the nature of the association between AS and PAH is currently unknown. Although two reports have noted a high incidence of PH in patients with AS $(9,10)$, these two studies used only echocardiography as diagnostic tools and all study participants were Russian. The diagnostic weakness of these reports may be related to the lack of validation using right heart catheterization and serial follow-up. The present patient was proven to have both AS and PAH, and the diagnosis of PAH was confirmed on both echocardiography and right heart catheterization.

In conclusion, the present patient was confirmed to have both AS and PAH. Although he initially reported lower back pain, the symptoms remained under control following analgesic treatment (sulfasalazine and meloxicam). Therefore, a diagnosis of drug-associated PAH is unlikely in this case. Due to the vague nature of the symptoms on presentation, it is necessary to have a high index of suspicion in order to make an early diagnosis of PAH. Doppler echocardiography is a good screening method, the results of which should be confirmed via right heart catheterization. However, further large-scale epidemiologic studies regarding the incidence of PAH among patients with AS are needed to elucidate the actual relationship between these two conditions.

The authors state that they have no Conflict of Interest (COI).

\section{References}

1. Simonneau G, Robbins IM, Beghetti M, et al. Updated clinical classification of pulmonary hypertension. J Am Coll Cardiol 54: S 43-S54, 2009.

2. Badesch DB, Champion HC, Sanchez MA, et al. Diagnosis and assessment of pulmonary arterial hypertension. J Am Coll Cardiol 54: S55-S66, 2009.

3. Phung S, Strange G, Chung LP, et al. Prevalence of pulmonary arterial hypertension in an Australian scleroderma population: screening allows for earlier diagnosis. Intern Med J 39: 682-691, 2009.

4. Launay D, Mouthon L, Hachulla E, et al. Prevalence and characteristics of moderate to severe pulmonary hyper tension in systemic sclerosis with and without tinterstitial lung disease. J Rheumatol 34: 1005-1011, 2007.

5. Chung L, Liu J, Parsons L, et al. Characterization of connective tissue disease-associated pulmonary arterial hypertension from REVEAL: identifying systemic sclerosis as a unique phenotype. Chest 138: 1383-1394, 2010.

6. Condliffe R, Kiely DG, Peacock AJ, et al. Connective tissue disease-associated pulmonary arterial hypertension in the modern treatment era. Am J Respir Crit Care Med 179: 151-157, 2009.

7. Humbert M, Sitbon O, Chaouat A, et al. Pulmonary arterial hypertension in France: results from a national registry. Am J Respir Crit Care Med 173: 1023-1030, 2006.

8. Shirai Y, Yasuoka H, Okano Y, Takeuchi T, Satoh T, Kuwana M. Clinical characteristics and survival of Japanese patients with connective tissue disease and pulmonary arterial hypertension: a single-centre cohort. Rheumatology 51: 1846-1854, 2012.

9. Karoli NA, Rebrov AP. [Pulmonary hypertension, involvement of the right and left cardiac parts in patients with ankylosing spondylarthritis]. Klin Med (Mosk) 82: 31-34, 2004 (in Russian, Abstract in English).

10. Poddubny $\breve{~ D A, ~ R e b r o v ~ A P . ~[P u l m o n a r y ~ h y p e r t e n s i o n ~ i n ~ p a t i e n t s ~}$ with ankylosing spondilitis: main factors of development]. Ter Arkh 80: 72-75, 2008 (in Russian, Abstract in English).

11. eHealthMe. Would you have Pulmonary hypertension when you have Ankylosing spondylitis? [Internet]. [cited 2014 March 9]. Available from: http://www.ehealthme.com/cs/ankylosing+spondyli tis/pulmonary+hypertension

12. Palazzi C, Salvarani C, D’Angelo S, Olivieri I. Aortitis and periaortitis in ankylosing spondylitis. Joint Bone Spine 78: 451-455, 2011.

13. Launay D, Hachulla E, Hatron PY, Jais X, Simonneau G, Humbert M. Pulmonary arterial hypertension: a rare complication of primary Sjögren syndrome: report of 9 new cases and review of the literature. Medicine (Baltimore) 86: 299-315, 2007.

(C) 2015 The Japanese Society of Internal Medicine http://www.naika.or.jp/imonline/index.html 